\title{
Downregulation of CD44 Inhibits Proliferation, Invasion and Migration of Osteosarcoma Cells by Regulating the Expression of Cathepsin S
}

Lingwei Kong ( $\nabla$ konglingwei0408@126.com )

Chengde Medical University Affiliated Hospital

Hairu Ji

Chengde Medical College: Chengde Medical University

Xintian Gan

Chengde Medical University Affiliated Hospital

Sheng Cao

Chengde Medical University Affiliated Hospital

Zhehong Li

Chengde Medical University Affiliated Hospital

Yu Jin

Chengde Medical University Affiliated Hospital

\section{Research Article}

Keywords: Osteosarcoma, CD44, cathepsin S, proliferation, migration, invasion

Posted Date: October 4th, 2021

DOl: https://doi.org/10.21203/rs.3.rs-943715/v1

License: (9) (i) This work is licensed under a Creative Commons Attribution 4.0 International License. Read Full License 


\section{Abstract}

\section{Background}

Osteosarcoma (OS) is a malignant bone tumour of mesenchymal origin. These tumours are characterised by rich vascularisation, therefore promoting rapid proliferation and facilitating metastasis. CD44 has been reported to be involved in OS, but its role and molecular mechanisms in the pathogenesis of the disease are not fully determined.

\section{Methods}

In this study, we investigated the antitumor effect of CD44 on the development of OS and further explored the molecular mechanisms. The expression of CD44, cathepsin S and MMP-9 was detected by Western blot (WB) and reverse transcription-polymerase chain reaction (RT-qPCR) in different cell lines (MG63, U2OS OS and hFOB 1.19). To elucidate the role of CD44 in OS, MG63 and U2OS cells were treated with small interference RNA (siRNA) to knock down CD44, and the knockdown efficiency was validated with GFP and RT-qPCR. Furthermore, cell proliferation was assayed using Cell Counting Kit-8 (CCK-8) and colony formation assays, and cell migration and invasion were assayed by transwell and wound-healing assays.

Results

We found that CD44 expression in the MG63 and U2OS OS cell lines was markedly increased compared to that of the human osteoblast hFOB 1.19 cell line. Knockdown of CD44 inhibited proliferation, migration, and invasion of MG63 and U2OS cells, possibly by regulating the expression of cathepsin $\mathrm{S}$ in OS.

\section{Conclusion}

Taken together, our data reinforced the evidence that CD44 knockdown inhibited cell proliferation, migration, and invasion of OS cells accompanied by altered expression of cathepsin $\mathrm{S}$. These findings offer new clues for OS development and progression, suggesting CD44 as a potential therapeutic target for OS.

\section{Introduction}

Osteosarcoma (OS) is the most common primary bone tumour, mainly occurring in children and adolescents, and the third most frequent in adults, following chondrosarcoma and chordoma. The overall incidence of OS is 3.4 per million cases per year worldwide [1], and the principal cause of death in patients suffering from OS is pulmonary metastasis [2]. Osteosarcoma is a primary bone cancer characterised by cancer cells that produce calcified osteoid extracellular matrix and inducing lung metastases with a high frequency. Despite recent advances in treating osteosarcoma with a combination of chemotherapy and surgery, the 5-year survival rate remains low, and the prognosis for patients is 
poor [3]. The cellular and molecular mechanisms underlying the progression of osteosarcoma, including the rate of cancer cell proliferation, the formation of metastatic lesions and the development of drug resistance, remain unclear [4].

Cluster of differentiation 44 (CD44) is a complex transmembrane adhesion glycoprotein considered an essential bridge molecule as it links the extracellular matrix and intracellular skeletal proteins and participates in intracellular signal transduction, affecting cell deformation or movement through cytoskeletal changes [5]. Numerous studies have reported that CD44 not only participates in normal cellular functions but also plays pivotal roles in pathological processes [6]. For example, CD44-RhoA-YAP signalling mediates mechanics-induced fibroblast activation, and targeting this pathway could ameliorate crystalline silica-induced silicosis and provide a potential therapeutic strategy to mitigate fibrosis [7]. It is noteworthy that CD44 expression was found upregulated in different tumours [8-10], promoting cancer cell invasion and migration $[11,12]$. However, the role and molecular mechanisms of CD44 in the development and progression of OS remain uncertain.

The present study analysed the CD44 expression pattern in OS cell lines using reverse transcription-quantitative PCR (RT-qPCR) and Western blot (WB). Furthermore, loss-of-function experiments were performed to investigate the biological roles of CD44 in OS. The results revealed that CD44 expression was upregulated in OS cell lines. In addition, in vitro assays revealed that CD44 downregulation inhibited cell proliferation, migration, and invasion, probably by regulating cathepsin $\mathrm{S}$. These findings suggest that CD44 functions as an oncogene and future research may contribute to the development of new tools for the diagnosis and treatment of OS.

\section{Materials And Methods}

\section{Cell culture}

The human OS MG63 and U2OS cell lines were purchased from the Cell Bank of Shanghai Institute of Cell Biology (Shanghai, China) and cultured in modified Eagle's medium (MEM, Gibco) supplemented with $10 \%$ fetal bovine serum (FBS, Gibco) at $37^{\circ} \mathrm{C}$ with $5 \% \mathrm{CO}_{2}$.

The normal human osteoblastic cell line hFOB 1.19 was purchased from the Cell Bank of Shanghai Institute of Cell Biology (Shanghai, China) and maintained in D-MEM/F-12 (Gibco) supplemented with $10 \%$ FBS (Gibco) and $0.3 \mathrm{mg} / \mathrm{mL}$ Geneticin (G418; Gibco) at $37^{\circ} \mathrm{C}$ with $5 \% \mathrm{CO}_{2}$.

\section{Small interference RNA transfection}

Small interference RNA (siRNA) for transfection were purchased from Ribobio (Guangzhou, China). Transfections ( $50 \mathrm{nM}$ final concentration of siRNA) were performed using Invitrogen Lipofectamine 2000 (Thermo Fisher Scientific) following the protocols of the manufacturer. Three different siRNAs (si-CD441】si-CD44-2, si-CD44-3) were tested, and si-CD44-1 and si-CD44-2 were selected for subsequent experiments. A control siRNA (si-NC) was used in all the experiments. 


\section{RT-qPCR analysis}

MG63 and U2OS cells were treated with si-CD44 or si-NC for $24 \mathrm{~h}$, and total RNA was extracted from the OS cell lines and the normal human osteoblastic cell line hFOB 1.19 using Trizol (Supersmart, China). Next, $2 \mu \mathrm{g}$ of RNA was used to synthesise the complementary DNA (CDNA) by reverse transcriptase (ABclonal, China). The resulting complementary cDNA was used for PCR analysis. The relative levels of genes were detected by RT-qPCR using SYBR Premix Ex Taq ${ }^{\text {TM }}$ (ABclonal, China). The PCR cycling conditions were $95^{\circ} \mathrm{C}$ for $5 \mathrm{~min}$, followed by denaturation for $10 \mathrm{sec}$ at $95^{\circ} \mathrm{C}$ and extension for $20 \mathrm{sec}$ at $60^{\circ} \mathrm{C}$ for 40 cycles. GAPDH was used as an internal loading control. All reactions were performed in triplicates. Fold changes were calculated using the $2-\triangle \triangle C \mathrm{Cq}$ method. The primers were as follows: $C D 44$

forward, 5'-GAGCAGCACTTCAGGAGGTT-3' and reverse, 5'-TGGTTGCTGTCTCAGTTGCT-3'; cathepsin $S$ forward, 5'-GCAGTGGCACAGTTGCATAA-3' and reverse, 5'-AGCACCACAAGAACCCATGT-3'; GAPDH forward, 5'-GTCTCCTCTGACTTCAACAGCG-3' and reverse, 5'-ACCACCCTGTTGCTGTAGCCAA-3'.

\section{Western blot analysis}

MG63 and U20S cells were treated with si-CD44 or si-NC for $48 \mathrm{~h}$, and total proteins were extracted using RIPA buffer containing protease inhibitor cocktail. Protein concentrations were determined using the BCA Protein Assay (Multi sciences). Proteins ( $30 \mu \mathrm{g} /$ lane) were separated by $10 \%$ SDS-PAGE and transferred to PVDF membranes. The membranes were blocked with $5 \%$ non-fat milk for $2 \mathrm{~h}$ at room temperature (RT). Next, the membranes were incubated with anti-CD44 (1:2000, ABclonal, China), cathepsin S (1:2000, Affinity, China), and MMP-9 (1:2000, ABclonal, China) at $4{ }^{\circ} \mathrm{C}$ overnight. Subsequently, the appropriate horseradish peroxidase (HRP)-linked secondary antibodies (1:5000, Sera care) were used to visualise the immunoreactivity. GAPDH was used as an internal control. The intensity of each band was measured with Image $\mathrm{J}$.

\section{Cell Counting Kit-8 (CCK-8) assay}

MG63 and U2OS cells were treated with si-CD44 or si-NC for $24 \mathrm{~h}$. Cells were prepared into suspension and MG-63, and U2OS cells were seeded in 96-well plates at a density of $1 \times 10^{3}$ cells per well and incubated in a humidified incubator at $37^{\circ} \mathrm{C}$ for $24,48,72 \mathrm{~h}$ and $96 \mathrm{~h}$. Subsequently, the cells were incubated with $10 \mu \mathrm{l} \mathrm{CCK-8} \mathrm{solution} \mathrm{for} \mathrm{another} 1 \mathrm{~h}$ at $37^{\circ} \mathrm{C}$. Optical density (OD) was determined at a wavelength of $450 \mathrm{~nm}$.

\section{Colony formation assay}

MG63 and U20S cells were treated with CD44 siRNA or negative control for $24 \mathrm{~h}$. Cells were then resuspended and seeded in 6-well plates at a density of 500 cells per well and cultured for 15 days. Subsequently, cells were fixed with pre-cooled methanol for $30 \mathrm{~min}$ at RT and stained with $0.1 \%$ crystal violet for 20 min at RT, washed twice with PBS and twice with double distilled water. The colonies were counted and analysed under a light microscope. 


\section{Wound-healing assay}

To evaluate the role of CD44 in OS cell migration, MG63 and U2OS cells were transfected with si-CD44 or si-NC for $24 \mathrm{~h}$. Cells were resuspended and seeded in 6-well plates at a density of $1 \times 10^{6}$ cells per well, and $2 \mathrm{ml}$ of culture medium supplemented with $10 \%$ FBS was added. Cells were grown to $90 \%$ confluence, and then a uniform and consistent wound was scraped on the bottom of the 6-well plate with a $200 \mu \mathrm{L}$ plastic pipette tip (time set as $0 \mathrm{~h}$ ). PBS was used to remove floating cells. Subsequently, cells were incubated in fresh complete medium ( $1 \%$ FBS) for 0,24 and $48 \mathrm{~h}$ and the number of migrated cells were observed and counted under a light microscope.

\section{Transwell assay}

Migration and invasion abilities of MG-63 and U2OS cells were measured using a transwell assay. The Matrigel was incubated at $37^{\circ} \mathrm{C}$ for $5 \mathrm{~h}$ before testing. OS cells were transfected with si-CD 44 or si-NC for $24 \mathrm{~h} .1 \times 10^{5}$ transfected cells were resuspended in serum-free medium and seeded in the upper chamber with or without Matrigel (BD Biosciences) for the invasion and migration assays, respectively. Subsequently, medium containing 20\% FBS was added to the lower chambers. Following a $24 \mathrm{~h}$ incubation, the cells from the upper compartments were scraped off with cotton swabs, while the cells that migrated to or invaded the lower surface of the membrane were fixed with pre-cooled methanol at RT for $20 \mathrm{~min}$ and stained with $0.1 \%$ crystal violet at RT for $20 \mathrm{~min}$. The stained cells were counted in five random fields off view under a light microscope at x200 magnification, and all experiments were repeated three times.

\section{Statistical analysis}

The results are presented as the mean \pm SD. Statistical analyses were performed using SPSS 23.0 (IBM Corp, USA) and GraphPad Prism 9.0 (La Jolla, CA, USA) software. ANOVA test was applied to compare differences among multiple groups. $\mathrm{P}<0.05$ was considered to indicate a statistically significant difference.

\section{Results}

1. CD44 is upregulated in OS cell lines. The present study first examined CD44 expression levels in the MG63, U2OS, and hFOB 1.19 cell lines by WB and RT-qPCR. Compared with the hFOB 1.19 cell line, CD44 expression was markedly upregulated in the OS cell lines (Fig. 1A-C).

2. CD44 knockdown in MG63 and U2OS cells in vitro. MG63 and U2OS cells were transfected with si$\mathrm{CD} 44$ or si-NC for $24 \mathrm{~h}$, and the transfection efficiency was detected using fluorescence microscopy (Fig. 2A). CD44 mRNA and protein expression was quantified by RT-PCR and Western blot, respectively, after CD44 knockdown in MG63 and U2OS cells. As shown in Figure 2B and C, the results revealed that the siRNA transfection decreased CD44 expression, but as expected, no significant difference was observed between the si-NC and control groups. For subsequent 
experiments, two (siCD44-1, siCD44-2) of the three CD44 siRNA with high transfection efficiency were selected.

3. CD44 knockdown inhibited the proliferation of MG63 and U2OS cells. To assess the role of CD44 in MG63 and U2OS cell proliferation, siRNA was transfected to silence CD44 expression. Subsequently, cell proliferation was assessed using CCK- 8 and colony formation assays. As demonstrated by the result of the CCK-8 assay, cell growth was suppressed in CD44-silenced MG63 and U2OS cells compared with the si-NC-transfected cells (Fig. 3A). In addition, the colony formation ability of si-CD44-transfected cells was decreased (Fig. 3B). These results revealed that downregulation of CD44 markedly decreased the proliferation of MG63 and U2OS cells.

4. CD44 knockdown inhibited the migration and invasion of MG63 and U2OS cells. To investigate the role of CD44 in the migration and invasion of OS cells, the wound-healing assay were used at 0,12 , or $24 \mathrm{~h}$ after transfection. The result of the wound-healing assay showed that the migration distances of cells transfected with si-NC was compared to the migration distances in CD44-silenced cells (Fig. 4A). The result of the transwell migration and invasion assay showed that the number of control si-NC-transfected cells was more than the number of CD44-silenced cells (Fig. 4B). Furthermore, Western blot was applied to evaluate the matrix metalloproteinase MMP-9 protein levels. As shown in Figure 4C, silencing of CD44 decreased MMP-9 expression in MG-63 and U2OS cells compared with the si-NC group. Therefore, the results suggested that the migration and invasion abilities of MG-63 and U20S cells were suppressed following CD44 knockdown.

5. CD44 knockdown downregulated the expression of cathepsin S in MG63 and U2OS cells. To further confirm the underlying mechanism of CD44 in OS, we detected the mRNA and protein expression of cathepsin S by RT-PCR and Western blot after CD44 knockdown in MG63 and U2OS cells. The mRNA and protein levels of cathepsin $S$ in the CD44-silenced OS cells were markedly reduced compared with the control cells (si-NC) at 24 and $48 \mathrm{~h}$ after transfection $(\mathrm{p}<0.01)$ (Fig. 5A-5B). These data indicated that CD 44 exerted its effects in $\mathrm{OS}$ in part by regulating cathepsin $\mathrm{S}$.

\section{Discussion}

The current treatment for osteosarcoma is surgical resection and combined neoadjuvant chemotherapy, which has increased the 5-year overall survival rate for osteosarcoma patients from less than $20 \%$ to more than $60 \%$ [13]. However, survival rates for patients with metastatic or recurrent osteosarcoma have remained virtually unchanged over the past 30 years, with an overall 5 -year survival rate as low as $20 \%$ [ 3 , 14]. The molecular mechanisms underlying the development of OS have not been fully explored. Therefore, it is crucial to elucidate the predictive markers of OS and their potential regulatory mechanisms.

CD44, also known as homing cell adhesion molecule, is a cell surface transmembrane glycoprotein molecule involved in cell-cell and cell-extracellular matrix communication. In humans, CD44 proteins are encoded by a highly conserved gene located on the short arm of chromosome 11 (11p13) [15]. CD44 can affect cell growth, proliferation and motility through changes in the cytoskeleton, as well as being 
involved in intracellular signal transduction [16]. Therefore, CD44 is a critical bridging molecule between the extracellular matrix and intracellular skeletal proteins.

CD44 expression is elevated in a wide range of malignant tumours [17], such as colon tumours [18], ovarian clear cell carcinoma [19], and glioblastoma [20]. Overexpression of CD44 splice mesenchymal isoform in OS cells induced EMT and invasion, followed by the gain of stem-like characteristics and chemoresistance [21]. In gastric cancer, CD44V6 regulates the transformation of normal mucosal epithelial cells into tumour cells and is associated with gastric cancer differentiation, lymph node metastasis, and pathological staging [22]. CD44 potentiates AKT activation to induce phosphorylation and nuclear translocation of $\mathrm{Mdm} 2$, which terminates the p53 genomic surveillance response. This allows DNA-damaged hepatocytes to escape p53-induced death and go on to become HCC progenitors [23]. We confirmed that CD44 is highly expressed in osteosarcoma cell lines compared to hFOB 1.19 human osteoblasts. Hence, we next evaluated the effects of CD44 on the proliferation, invasion, and migration of osteosarcoma cells.

We found that downregulation of CD44 inhibited the proliferation, invasion, and migration ability of osteosarcoma cells MG63 and U20S, which is consistent with the reported role of CD44 in most tumours $[8,10,17]$, suggesting that CD 44 may be a pro-oncogene in osteosarcoma.

To know more about the molecular mechanisms, we next investigated the expression of cathepsin S. Like matrix metalloproteinases, members of the histone family have been associated with metastasis and cancer recurrence [24]. Cathepsin S (CTSS), a member of the histone family of enzymes, is highly expressed in renal clear cell carcinoma [25], hepatocellular carcinoma [26], cervical cancer [27], lung cancer [28] and other tumours and is an essential regulator of tumour growth and invasion. Suppression of cell migration and invasion by modulation of $\mathrm{Ca}^{2+}$-dependent downstream effectors after CTSS inhibition [29]. The expression of CTSS was regulated by PI3K/Akt and Ras/Raf/MAPK signalling pathways, is a candidate target for blocking the metastasis of breast and oral cancers [30, 31]. However, it is not clear what role cathepsin S plays in osteosarcoma. Previous experiments found that cathepsin $S$ was highly expressed in osteosarcoma cells and that silencing inhibited the invasion and metastasis of osteosarcoma cells.

Previous studies have reported that CD44 can promote tumour stem cell properties in triple-negative breast cancer by regulating the PI3K/AKT pathway [32]. Interaction of hyaluronan and CD44 enhances neutrophil phagocytosis and IL-8 production via the p38 and ERK1/2-MAPK signalling pathways [33]. Activation of the receptor complex CD74/CD44 can lead to activation of the ERK1/2, PI3K-Akt signalling cascade, NFKB and AMP-activated protein kinase (AMPK) pathways [34]. Therefore, we speculate that CD44 may exert its biological effects on osteosarcoma cells through the regulation of cathepsin $\mathrm{S}$. The experimental results showed that cathepsin S expression was downregulated after CD44 silencing compared to the si-NC group. Therefore, we suggest that CD44 may exert regulation of osteosarcoma cell proliferation, invasion, and metastasis through the regulation of cathepsin S. However, the specific 
regulatory mechanisms of the two still need to be further explored, and this will be the focus of our subsequent work.

\section{Conclusion}

This study shows that inhibition of CD44 attenuates cell proliferation, migration, and invasion, possibly by regulating the expression of cathepsin $\mathrm{S}$ in OS cells. These findings suggest that CD44 may be an oncogenic factor in the progression of OS and may be a promising molecular marker for the diagnosis and treatment of OS.

\section{Abbreviations}

OS: Osteosarcoma; CD44: cluster of differentiation 44; CCK8: Cell Counting Kit-8; MMP9: Matrix metalloproteinase 9; WB: Western blot; RT-qPCR: reverse transcription-polymerase chain reaction; siRNA: small interference RNA

\section{Declarations}

\section{Acknowledgements}

Thank you for medical science research project in Hebei province $(20200352,20200378)$ and natural science research youth fund for higher education in Hebei province (QN2020107) support.

\section{Authors' contributions}

Lingwei Kong was responsible for manuscript writing and experiment conducting; Hairu Ji and Xintian Gan contributed to data collection and analysis; Sheng Cao collected the literature and explained the results; Yu Jin designed this study and reviewed this article. The authors read and approved the final manuscript.

\section{Funding}

This work was supported by medical science research project in Hebei province $(20200352,20200378)$ and natural science research youth fund for higher education in Hebei province (QN2020107).

\section{Availability of data and materials}

The datasets supporting the conclusions of this article are included within the article.

\section{Declarations}

Ethics approval and consent to participate Not applicable.

\section{Consent for publication}


Not applicable.

\section{Competing interests}

The authors report no declarations of interest.

\section{References}

[1] A.C. da Costa, F. Santa-Cruz, L.A.R. Mattos, M.A. Rego Aquino, C.R. Martins, A.A. Bandeira Ferraz, J.L. Figueiredo, Cathepsin S as a target in gastric cancer, Mol Clin Oncol 12(2) (2020) 99-103.

[2] M.F. Heymann, F. Lézot, D. Heymann, The contribution of immune infiltrates and the local microenvironment in the pathogenesis of osteosarcoma, Cellular immunology 343 (2019) 103711.

[3] L. Marchandet, M. Lallier, C. Charrier, M. Baud'huin, B. Ory, F. Lamoureux, Mechanisms of Resistance to Conventional Therapies for Osteosarcoma, Cancers (Basel) 13(4) (2021).

[4] K.H. Lu, R.C. Lin, J.S. Yang, W.E. Yang, R.J. Reiter, S.F. Yang, Molecular and Cellular Mechanisms of Melatonin in Osteosarcoma, Cells 8(12) (2019).

[5] G.T. Lim, D.G. You, H.S. Han, H. Lee, S. Shin, B.H. Oh, E.K.P. Kumar, W. Um, C.H. Kim, S. Han, S. Lee, S. Lim, H.Y. Yoon, K. Kim, I.C. Kwon, D.G. Jo, Y.W. Cho, J.H. Park, Bioorthogonally surface-edited extracellular vesicles based on metabolic glycoengineering for CD44-mediated targeting of inflammatory diseases, Journal of extracellular vesicles 10(5) (2021) e12077.

[6] Y. Dong, A.A. Arif, J. Guo, Z. Ha, S.S.M. Lee-Sayer, G.F.T. Poon, M. Dosanjh, C.D. Roskelley, T. Huan, P. Johnson, CD44 Loss Disrupts Lung Lipid Surfactant Homeostasis and Exacerbates Oxidized LipidInduced Lung Inflammation, Front Immunol 11 (2020) 29.

[7] S. Li, C. Li, Y. Zhang, X. He, X. Chen, X. Zeng, F. Liu, Y. Chen, J. Chen, Targeting Mechanics-Induced Fibroblast Activation through CD44-RhoA-YAP Pathway Ameliorates Crystalline Silica-Induced Silicosis, Theranostics 9(17) (2019) 4993-5008.

[8] R. Pothuraju, S. Rachagani, S.R. Krishn, S. Chaudhary, R.K. Nimmakayala, J.A. Siddiqui, K. Ganguly, I. Lakshmanan, J.L. Cox, K. Mallya, S. Kaur, S.K. Batra, Molecular implications of MUC5AC-CD44 axis in colorectal cancer progression and chemoresistance, Mol Cancer 19(1) (2020) 37.

[9] K.E. Gomez, F. Wu, S.B. Keysar, J.J. Morton, B. Miller, T.S. Chimed, P.N. Le, C. Nieto, F.N. Chowdhury, A. Tyagi, T.R. Lyons, C.D. Young, H. Zhou, H.L. Somerset, X.J. Wang, A. Jimeno, Cancer Cell CD44 Mediates Macrophage/Monocyte-Driven Regulation of Head and Neck Cancer Stem Cells, Cancer Res 80(19) (2020) 4185-4198.

[10] X. Liu, R. Taftaf, M. Kawaguchi, Y.F. Chang, W. Chen, D. Entenberg, Y. Zhang, L. Gerratana, S. Huang, D.B. Patel, E. Tsui, V. Adorno-Cruz, S.M. Chirieleison, Y. Cao, A.S. Harney, S. Patel, A. Patsialou, Y. Shen, S. 
Avril, H.L. Gilmore, J.D. Lathia, D.W. Abbott, M. Cristofanilli, J.S. Condeelis, H. Liu, Homophilic CD44 Interactions Mediate Tumor Cell Aggregation and Polyclonal Metastasis in Patient-Derived Breast Cancer Models, Cancer discovery 9(1) (2019) 96-113.

[11] H. Cao, J. Xiao, M.E. Reeves, K. Payne, C.S. Chen, D.J. Baylink, G. Marcucci, Y. Xu, Discovery of proangiogenic CD44+mesenchymal cancer stem cells in an acute myeloid leukemia patient's bone marrow, J Hematol Oncol 13(1) (2020) 63.

[12] T. Kong, R. Ahn, K. Yang, X. Zhu, Z. Fu, G. Morin, R. Bramley, N.C. Cliffe, Y. Xue, H. Kuasne, Q. Li, S. Jung, A.V. Gonzalez, S. Camilleri-Broet, M.C. Guiot, M. Park, J. Ursini-Siegel, S. Huang, CD44 Promotes PDL1 Expression and Its Tumor-Intrinsic Function in Breast and Lung Cancers, Cancer Res 80(3) (2020) 444457 .

[13] C. Zheng, F. Tang, L. Min, F. Hornicek, Z. Duan, C. Tu, PTEN in osteosarcoma: Recent advances and the therapeutic potential, Biochim Biophys Acta Rev Cancer 1874(2) (2020) 188405.

[14] J. Cui, D. Dean, F.J. Hornicek, Z. Chen, Z. Duan, The role of extracelluar matrix in osteosarcoma progression and metastasis, Journal of experimental \& clinical cancer research : CR 39(1) (2020) 178.

[15] A. Ouhtit, B. Rizeq, H.A. Saleh, M.M. Rahman, H. Zayed, Novel CD44-downstream signaling pathways mediating breast tumor invasion, International journal of biological sciences 14(13) (2018) 1782-1790.

[16] P. Govindaraju, L. Todd, S. Shetye, J. Monslow, E. Puré, CD44-dependent inflammation, fibrogenesis, and collagenolysis regulates extracellular matrix remodeling and tensile strength during cutaneous wound healing, Matrix biology : journal of the International Society for Matrix Biology 75-76 (2019) 314330 .

[17] C. Chen, S. Zhao, A. Karnad, J.W. Freeman, The biology and role of CD44 in cancer progression: therapeutic implications, J Hematol Oncol 11(1) (2018) 64.

[18] S.P.J. Joosten, M. Spaargaren, H. Clevers, S.T. Pals, Hepatocyte growth factor/MET and CD44 in colorectal cancer: partners in tumorigenesis and therapy resistance, Biochim Biophys Acta Rev Cancer 1874(2) (2020) 188437.

[19] Y. Yamada, T. Miyamoto, H. Kashima, H. Kobara, R. Asaka, H. Ando, S. Higuchi, K. Ida, T. Shiozawa, Lipocalin 2 attenuates iron-related oxidative stress and prolongs the survival of ovarian clear cell carcinoma cells by up-regulating the CD44 variant, Free radical research 50(4) (2016) 414-25.

[20] T.T. Lah, M. Novak, B. Breznik, Brain malignancies: Glioblastoma and brain metastases, Semin Cancer Biol 60 (2020) 262-273.

[21] R. Bhattacharya, T. Mitra, S. Ray Chaudhuri, S.S. Roy, Mesenchymal splice isoform of CD44 (CD44s) promotes EMT/invasion and imparts stem-like properties to ovarian cancer cells, J Cell Biochem 119(4) (2018) 3373-3383. 
[22] S. Liang, H.L. Li, G.Y. Han, J.H. Cui, CD44V6 regulates gastric carcinoma occurrence and development through up-regulating VEGF expression, European review for medical and pharmacological sciences $21(22)(2017)$ 5121-5128.

[23] D. Dhar, L. Antonucci, H. Nakagawa, J.Y. Kim, E. Glitzner, S. Caruso, S. Shalapour, L. Yang, M.A. Valasek, S. Lee, K. Minnich, E. Seki, J. Tuckermann, M. Sibilia, J. Zucman-Rossi, M. Karin, Liver Cancer Initiation Requires p53 Inhibition by CD44-Enhanced Growth Factor Signaling, Cancer cell 33(6) (2018) 1061-1077.e6.

[24] A. Steimle, H. Kalbacher, A. Maurer, B. Beifuss, A. Bender, A. Schafer, R. Muller, I.B. Autenrieth, J.S. Frick, A novel approach for reliable detection of cathepsin $\mathrm{S}$ activities in mouse antigen presenting cells, $\mathrm{J}$ Immunol Methods 432 (2016) 87-94.

[25] E. Song, W. Song, M. Ren, L. Xing, W. Ni, Y. Li, M. Gong, M. Zhao, X. Ma, X. Zhang, R. An, Identification of potential crucial genes associated with carcinogenesis of clear cell renal cell carcinoma, $\mathrm{J}$ Cell Biochem 119(7) (2018) 5163-5174.

[26] X. Wang, L. Xiong, G. Yu, D. Li, T. Peng, D. Luo, J. Xu, Cathepsin S silencing induces apoptosis of human hepatocellular carcinoma cells, American journal of translational research 7(1) (2015) 100-10.

[27] M.C. Hsin, Y.H. Hsieh, P.H. Wang, J.L. Ko, I.L. Hsin, S.F. Yang, Hispolon suppresses metastasis via autophagic degradation of cathepsin S in cervical cancer cells, Cell Death Dis 8(10) (2017) e3089.

[28] R. Brown, S. Nath, A. Lora, G. Samaha, Z. Elgamal, R. Kaiser, C. Taggart, S. Weldon, P. Geraghty, Cathepsin S: investigating an old player in lung disease pathogenesis, comorbidities, and potential therapeutics, Respir Res 21(1) (2020) 111.

[29] H.H. Lin, S.J. Chen, M.R. Shen, Y.T. Huang, H.P. Hsieh, S.Y. Lin, C.C. Lin, W.W. Chang, J.Y. Chang, Lysosomal cysteine protease cathepsin $\mathrm{S}$ is involved in cancer cell motility by regulating store-operated $\mathrm{Ca}(2+)$ entry, Biochim Biophys Acta Mol Cell Res 1866(12) (2019) 118517.

[30] M.J. Hsieh, C.W. Lin, M.K. Chen, S.Y. Chien, Y.S. Lo, Y.C. Chuang, Y.T. Hsi, C.C. Lin, J.C. Chen, S.F. Yang, Inhibition of cathepsin $\mathrm{S}$ confers sensitivity to methyl protodioscin in oral cancer cells via activation of p38 MAPK/JNK signaling pathways, Scientific reports 7 (2017) 45039.

[31] J. Gautam, Y.K. Bae, J.A. Kim, Up-regulation of cathepsin S expression by HSP90 and 5-HT7 receptordependent serotonin signaling correlates with triple negativity of human breast cancer, Breast Cancer Res Treat 161(1) (2017) 29-40.

[32] J. Bai, W.B. Chen, X.Y. Zhang, X.N. Kang, L.J. Jin, H. Zhang, Z.Y. Wang, HIF-2a regulates CD44 to promote cancer stem cell activation in triple-negative breast cancer via PI3K/AKT/mTOR signaling, World journal of stem cells 12(1) (2020) 87-99. 
[33] C.H. Lu, C.H. Lin, K.J. Li, C.Y. Shen, C.H. Wu, Y.M. Kuo, T.S. Lin, C.L. Yu, S.C. Hsieh, Intermediate Molecular Mass Hyaluronan and CD44 Receptor Interactions Enhance Neutrophil Phagocytosis and IL-8 Production via p38- and ERK1/2-MAPK Signalling Pathways, Inflammation 40(5) (2017) 1782-1793.

[34] H. Su, N. Na, X. Zhang, Y. Zhao, The biological function and significance of CD74 in immune diseases, Inflamm Res 66(3) (2017) 209-216.

\section{Figures}

A

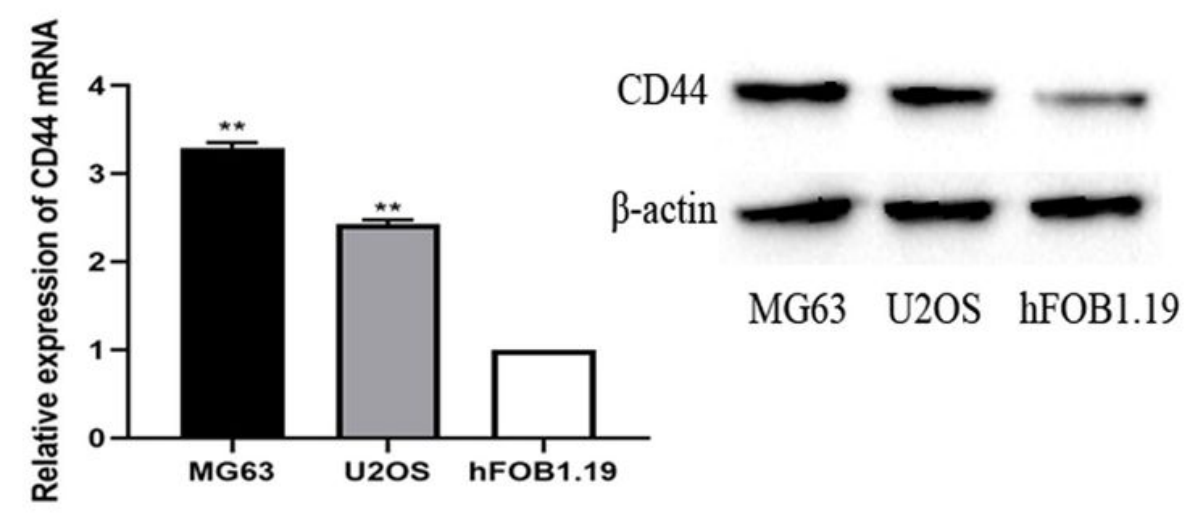

$\mathrm{C}$

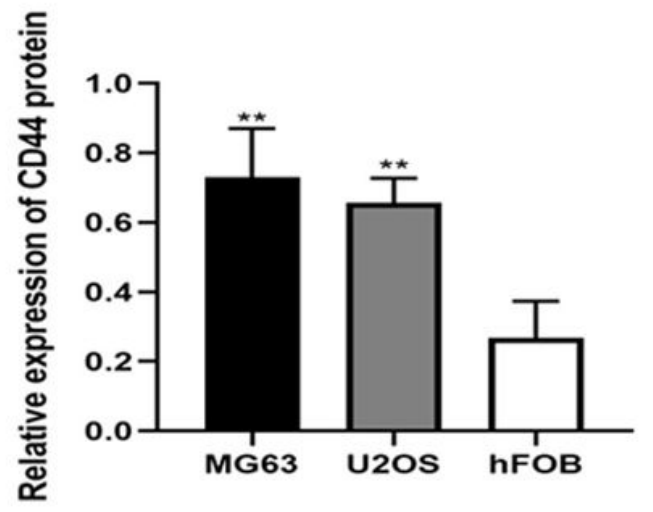

Figure 1

CD44 is upregulated in OS cell lines. (A) CD44 mRNA levels in MG63, U2OS, and hFOB 1.19 cell lines. **P $<0.01$ vs. hFOB group. (B) and (C) CD44 protein levels in MG63, U2OS, and hFOB 1.19 cell lines. ${ }^{*} \mathrm{P}<$ 0.01 vs. hFOB group. 


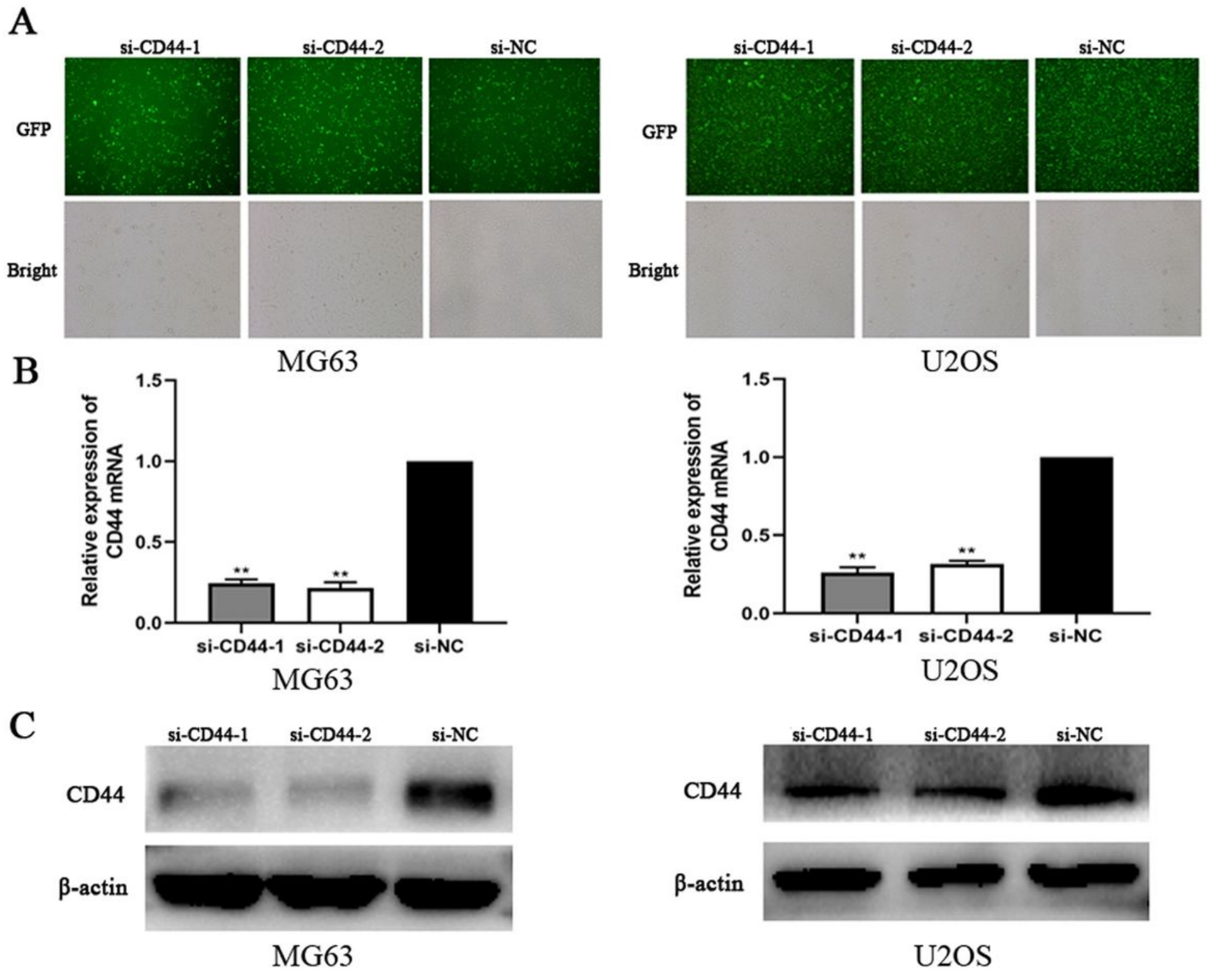

Figure 2

CD44 knockdown in MG63 and U20S cells in vitro. (A) Transfection efficiency of MG-63 and U2OS cells was assessed by fluorescence microscopy. GFP, green fluorescent protein. Magnification, x200. (B) Reverse transcription-quantitative PCR analysis was used to assess the mRNA expression levels of CD44 in MG-63 and U20S cells after transfection for $24 \mathrm{~h}$. ${ }^{*} \mathrm{P}<0.01$ vs. si-NC group. (C) Western blot was used to assess CD44 expression levels in MG-63 and U2OS cells $48 \mathrm{~h}$ after transfection. 


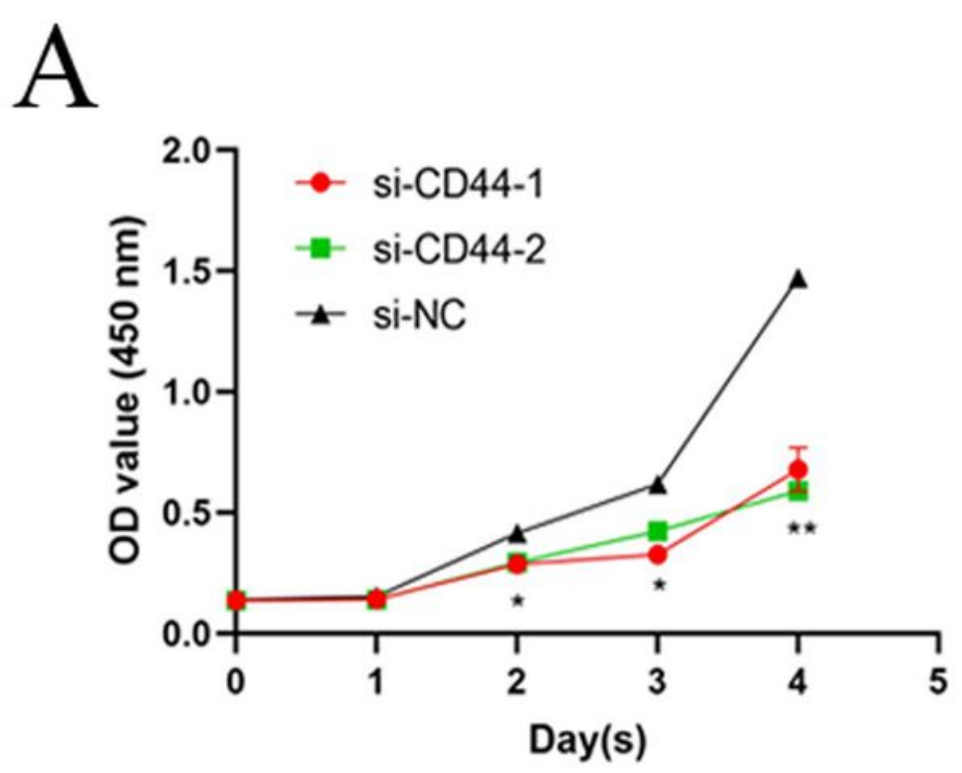

MG63

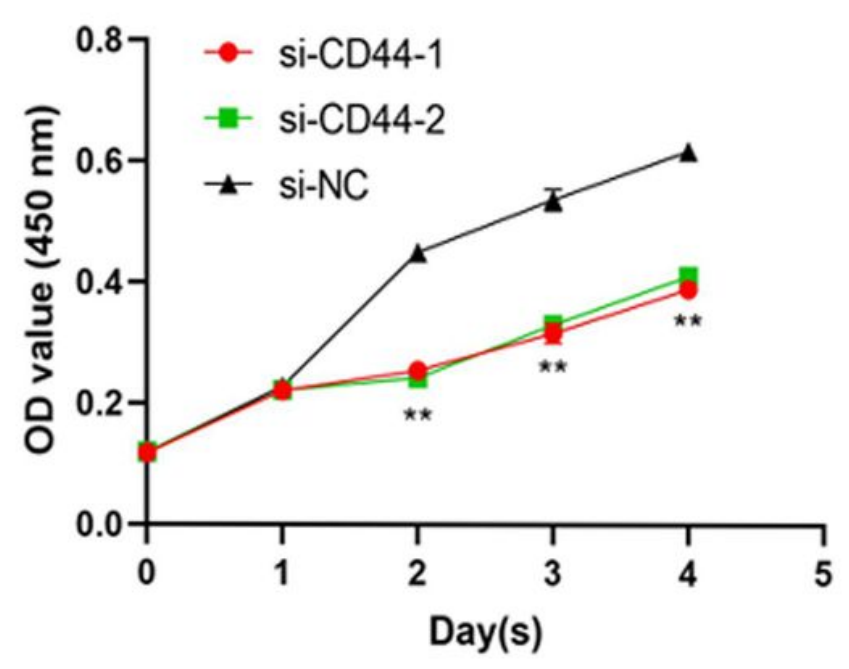

U2OS

B
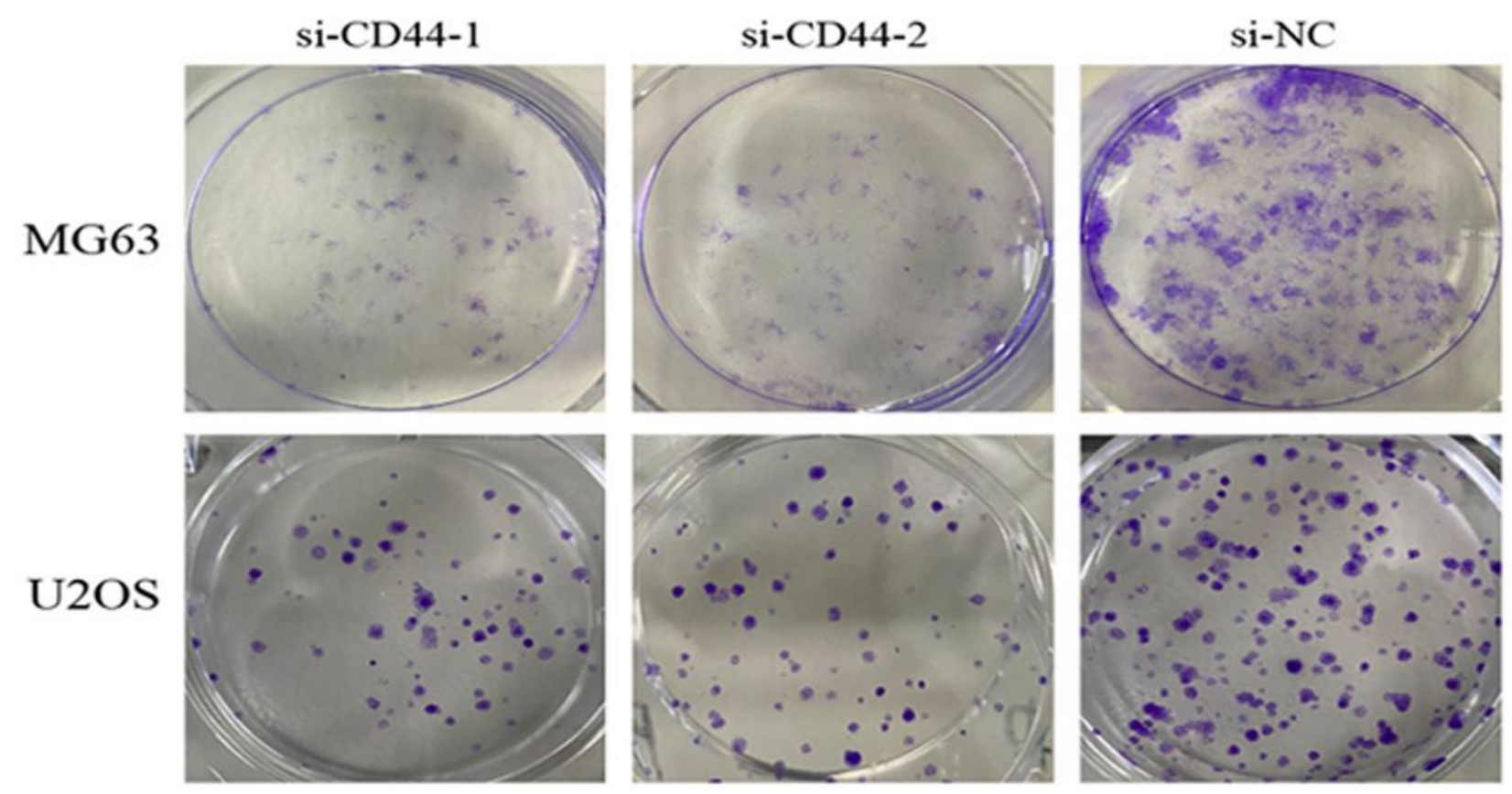

Figure 3

CD44 knockdown inhibited the proliferation of MG63 and U20S cells. (A) Cell proliferation and (B) colony formation in MG 63 and U2OS cells. All data are presented as the mean \pm SD of $n=3$ experiments ( ${ }^{\mathrm{P}}<$ $0.05, \star \star P<0.01$ vs. si-NC group). 
A
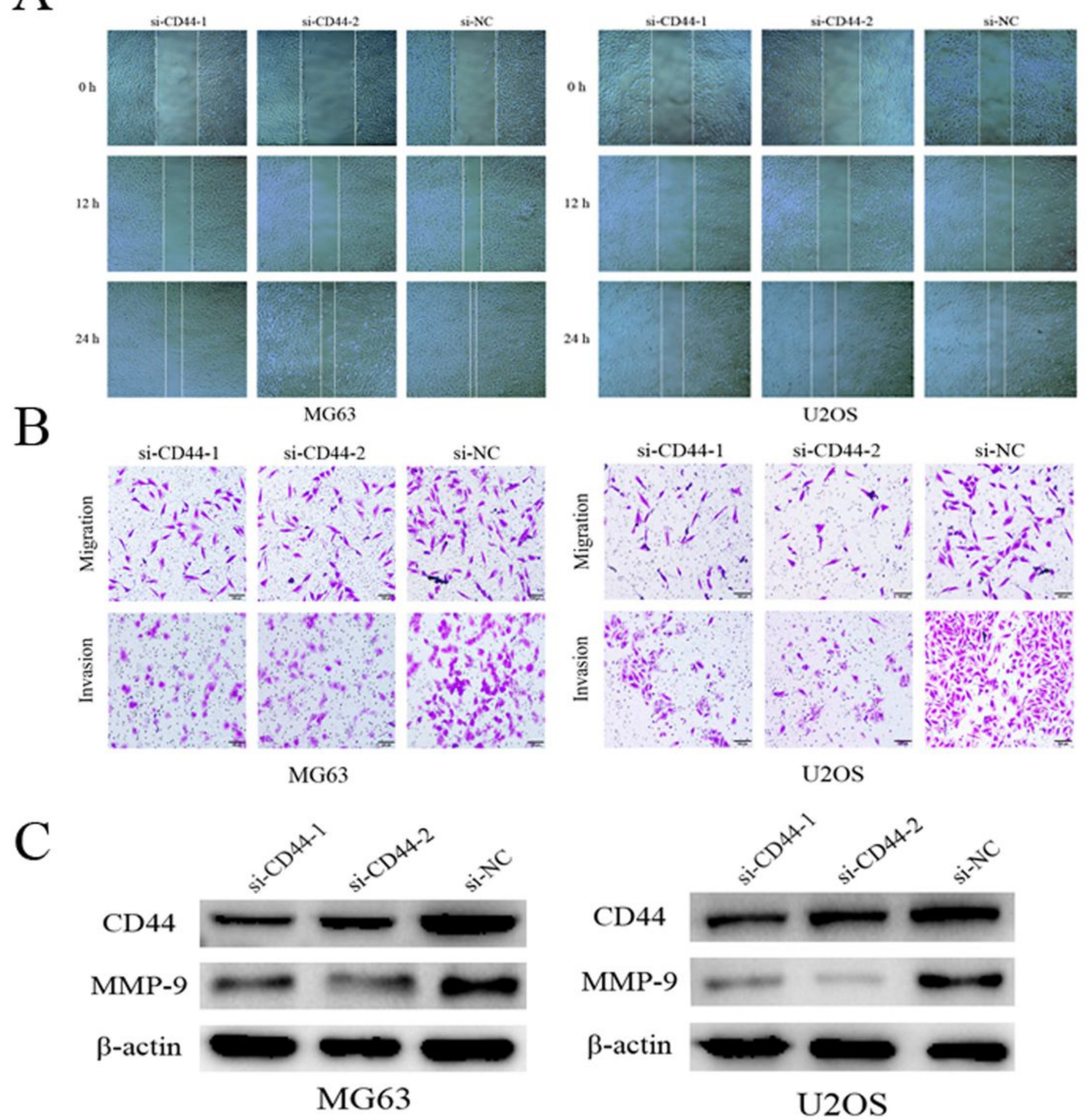

U2OS

\section{Figure 4}

CD44 knockdown inhibited the migration and invasion of MG63 and U2OS cells. (A) A wound-healing assay was performed to detect the migration of MG 63 and U2OS cells. (B) Transwell assay was performed to detect migration and invasion of MG 63 and U2OS cells. Magnification, $x 200$. (C) Expression levels of migration and invasion related proteins (MMP 9) were detected by Western blotting. 
A

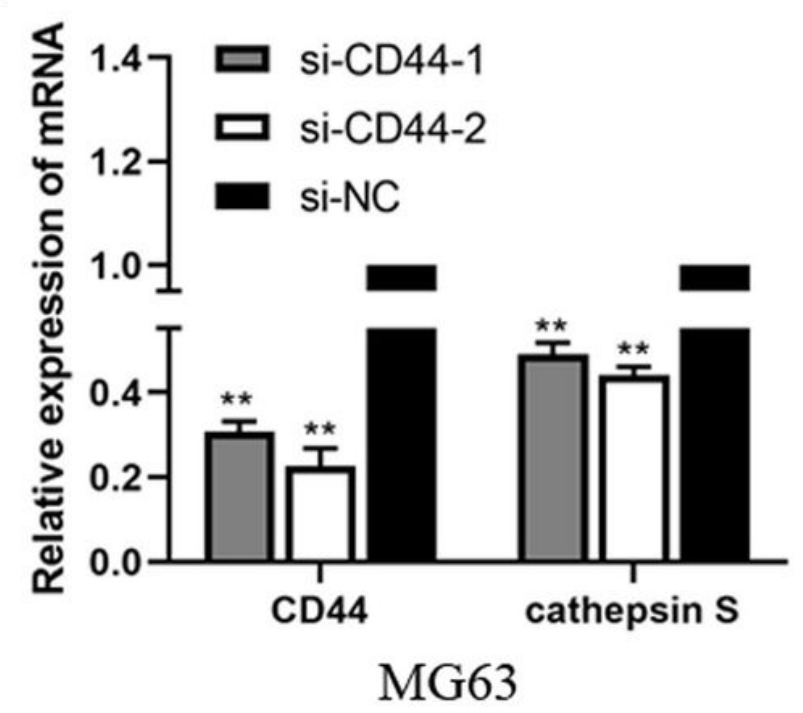

B

\section{CD44}
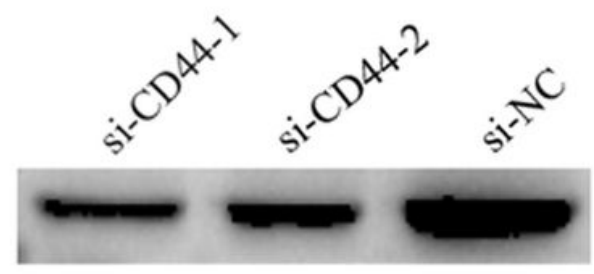

cathepsin S

$\beta$-actin
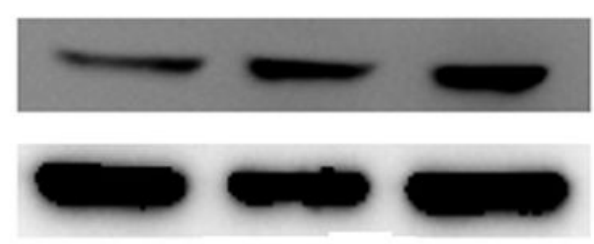

MG63

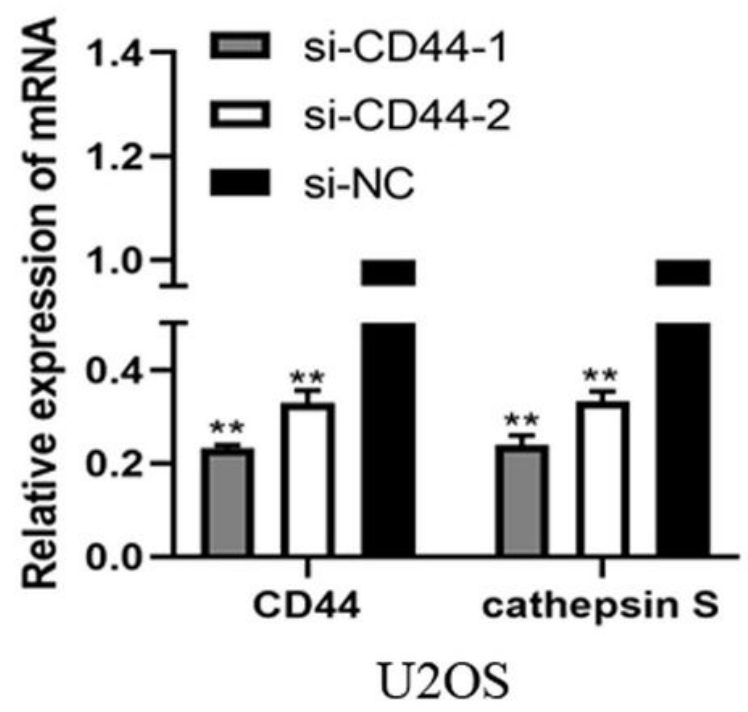

CD44

cathepsin S

$\beta$-actin

\section{Figure 5}

CD44 knockdown downregulated the expression of cathepsin S in MG63 and U2OS cells. (A) mRNA expression levels of CD44, cathepsin S, and $\beta$-actin were detected by RT-qPCR. (B) Protein expression levels of $C D 44$, cathepsin $S$, and $\beta$-actin were detected by Western blotting. ${ }^{*} P<0.01$ vs. control group. 